\title{
Anomalous scaling in depinning transitions
}

\author{
Onuttom Narayan \\ Department of Physics, University of California, Santa Cruz, CA 95064
}

(October 29, 2018)

\begin{abstract}
It is demonstrated that the renormalization group (RG) flows of depinning transitions do not depend on whether the driving force or the system velocity is kept constant. This allows for a comparison between RG results and corresponding self-organized critical models. However, close to the critical point, scaling functions cross over to forms that can have singular behavior not seen in equilibrium thermal phase transitions. These can be different for the constant force and constant velocity driving modes, leading to different apparent critical exponents. This is illustrated by comparing extremal dynamics for interface depinning with RG results, deriving the change in apparent exponents. Thus care has to be exercised in such comparisons.
\end{abstract}

PACS numbers: 68.35.Rh, 05.65.+b, 64.60.Ht, 05.45.-a

Driven dynamics of disordered systems have been studied extensively from two different approaches. As the external driving force is increased, a system undergoes a depinning transition from a macroscopically static state (with transient motion) to a moving steady state. This transition has been successfully described as a dynamical second order phase transition [1], and analyzed within the framework of the renormalization group (RG), originally for charge-density waves [2] and subsequently for interfaces [3] and a variety of other systems [4, 8]. This approach obtains the scaling behavior near the transition where the system starts to move. On the other hand, the concept of self-organized criticality (SOC), in the original sandpile model [5] and descendants thereof [6] has been used to obtain the behavior of systems that are forced to stay at the depinning transition.

One would expect there to be connections between these two viewpoints [7]; indeed, it is possible to map automaton models for CDWs to the original SOC model [8,9], and $1+1$ dimensional moving interface to a slightly different SOC model [10]. The connections between the two have been exploited to obtain the dynamical critical exponent $z$ for CDWs by borrowing from exact results for SOC sandpiles 11. However, it has been pointed out [6] that one has to be careful whether the depinning transition is approached with a time independent external driving force, tuned to its critical value, or with a time independent (infinitesimal) velocity. In the latter case, one envisages a feedback loop that adjusts the external force in a time-dependent way, so as to ensure that the rate at which the system moves (averaged over its entire spatial extent) is strictly time independent. It has been suggested [6] that the critical behavior at the depinning transition could be different for these two driving modes. As an extreme example of constant current driving, there is the class of "extremal models" [12 14, where at every (discrete) time step, there is activity only at one lattice site in the system.

For constant force driving, there are fluctuations in the (spatially averaged) velocity of the system, while for constant velocity driving there are fluctuations in the external force. In either case, at a non-zero driving rate, the fluctuations are small for sufficiently large systems, and thus the two driving mechanisms should be equivalent. However, the large system limit is problematic at the critical point. For instance, with constant force driving, the mean square fluctuations in the velocity of a large system are $(\delta v)^{2} \sim v^{2}(\xi / L)^{d}$, where $v$ is the mean velocity, $\xi$ is the correlation length at velocity $v$ (defined through the velocity autocorrelation function or other methods), $L$ is the linear extent of the system, and $d$ is its dimensionality. One would expect that holding the velocity constant by adjusting the force will make a qualitative difference to the dynamics when the velocity fluctuations are a significant fraction of the mean velocity, i.e. $\xi \sim L$. Heuristically, for a fixed $v$ and $L \rightarrow \infty$, imposing a constant velocity does not affect the dynamics, since the total 'activity' at every time step is $v L^{d}$, so that avalanches etc. are free to unfold with their own internal timescales. On the other hand, for fixed $L$ as $v \rightarrow 0$, imposing a constant velocity 'chokes off' the dynamics: avalanches proceed sequentially, with internal dynamics determined by the velocity constraint.

In this paper, we show that (to the extent that continuum descriptions are appropriate) both constant force and constant velocity driving actually have the same scaling under the renormalization group. However, scaling functions for various quantities, which can have different behavior in the limits $\xi<<L$ and $\xi>>L$, are often different in the $\xi>>L$ regime for the two driving modes. It is standard for scaling functions in equilibrium critical phenomena to behave differently when their arguments are small and large, but as will be discussed later, dynamical critical points have even more freedom in how scaling functions can behave. It is this freedom that allows identical RG flows to still yield different scaling functions for the two driving modes in the $\xi>>L$ regime.

We demonstrate this by focusing on a particular system, that of a interface with short range internal elastic interactions that is driven through a disordered medium 
under the influence of an external force. Due to the disorder, different parts of the interface experience different random pinning forces as they move forward. Using the $\mathrm{RG}$ relationship between the interface velocity and the correlation length [3] $v \sim \xi^{\zeta-z}$, we see that the crossover to $\xi>L$ occurs at $v \sim L^{\zeta-z}$. Note that extremal dynamics for a $d+1$ dimensional interface corresponds to $v \sim L^{-d}$, and $z-\zeta<d$ in all dimensions, so that $\xi>>L$ for large $L$.

It has been pointed out 15, 13, 16 that in extremal models for the motion of pinned interfaces, the roughness of the interface can scale anomalously. Thus for an $1+1$ dimensional interface in a system of size $L$ in steady state [17], if $h(x, t)$ is the interface position as a function of the transverse coordinate $x$ and the time $t$, the roughness $w(t)=\left[\left\langle\left\{h\left(x, t+t_{0}\right)-\bar{h}\left(t+t_{0}\right)-h\left(x, t_{0}\right)+\right.\right.\right.$ $\left.\left.\left.\bar{h}\left(t_{0}\right)\right\}^{2}\right\rangle\right]^{1 / 2}$ (all averages are spatial averages) has the scaling form 16, 18

$$
w(t)=t_{v}^{\beta} L^{-1 / 2} \varphi\left(\frac{L}{t^{1 / z_{v}}}\right) .
$$

(The subscripts on $\beta$ and $z$ denote constant velocity driving.) It can be shown [13,16 that the scaling function $\varphi$ goes to a constant for large values of its argument. The explicit $L$ dependence that remains is anomalous, contrary to the normal expectation of a well defined $L \rightarrow \infty$ limit. As a consequence of this, if the long time (steady state) roughness scales as $w(t \rightarrow \infty) \sim L^{\zeta}$, then the conventional relation $\zeta=z \beta$ is replaced with

$$
\zeta=z_{v} \beta_{v}-\frac{1}{2}
$$

With the additional result 13, 16, 19,

$$
z_{v}=1+\zeta
$$

one can obtain $\beta_{v}$ in terms of $\zeta$. Both Eq.(2) and Eq.(3) differ from renormalization group (RG) results [3] for an interface driven with a constant applied force. In particular, the RG analysis yields no simple relation between the dynamic exponent $z$ and the roughness exponent $\zeta$, unlike Eq.(3). Nor is there any reason to expect the anomalous scaling form of Eq.(11), and therefore $\zeta=z \beta$ should hold instead of Eq.(2).

We first demonstrate that, despite appearances, the RG flows are unaffected by going from a constant force driving mode to a constant velocity driving mode. The standard equation for zero-temperature driven dynamics of an interface is 20

$$
\partial_{t} h(x, t)=\nabla^{2} h(x, t)+Y(h(x, t) ; x)+F .
$$

Here $\nabla^{2} h(x, t)$ comes from the (short-ranged) elastic energy of the interface, $Y(h ; x)$ is a pinning force that comes from a random (impurity) potential, and $F$ is the external driving force. (Of course, it is not necessary that all lattice growth models - e.g. the Sneppen model [12] - can be mapped to this or any continuum equation.) We have neglected KPZ like terms 21]. Using the Martin Siggia Rose method 23 and introducing an auxiliary field $\hat{h}(x, t)$, one can construct [3] a generating functional $Z$ which can be written as

$$
\begin{aligned}
Z=\int[d h][d \hat{h}] \exp \left[\int d^{d} x d t\right. & \\
& \left.\quad i \hat{h}(x, t)\left\{\partial_{t} h-\nabla^{2} h-F-Y(h ; x)\right\}\right]
\end{aligned}
$$

where integrating out the auxiliary field yields a product of $\delta$-functions that imposes Eq.(4). If instead the interface is driven at constant velocity, Eq.(5) is replaced with

$$
\begin{gathered}
Z^{\prime}=\int[d F][d \mu][d h][d \hat{h}] \exp \left[\int d^{d} x d t\right. \\
\left.i \hat{h}(x, t)\left\{\partial_{t} h-\nabla^{2} h-F(t)-Y(h ; x)\right\}\right] \\
+i \mu(t)\left\{v-\partial_{t} h\right\}
\end{gathered}
$$

This extension is actually not difficult to understand: at any time $t$, by first integrating over $\mu(t)$ and $\hat{h}(x, t)$, we obtain $\delta$-function constraints that impose $\left\langle\partial_{t} h\right\rangle=v$ (the average here is a spatial average) and Eq.(5) with a (as yet unknown) driving force $F(t)$. Now performing the integral over $F$, the integral together with the first constraint sets $F(t)$ to be whatever it has to be for $\left\langle\partial_{t} h\right.$ to be equal to $v$. One is left with the second constraint, i.e. Eq.(4), with $F(t)$ adjusted to ensure constant velocity. Even though $F(t)$ is now a dynamical variable instead of a parameter, since it is not a field, i.e. it has no spatial dependence (nor has $\mu(t)$ ), when short distance fluctuations are eliminated under renormalization there are no extra loop corrections in $Z^{\prime}$ compared to $Z$.

As mentioned earlier, even though the RG fixed point is the same for constant force and constant velocity driving, the behavior of scaling functions are different for the two driving modes in the $\xi>>L$ regime. To illustrate this, we first consider the scaling of the duration of an avalanche as a function of its linear size. This has the general scaling form

$$
t(l, L, \xi)=l^{z} T(l / L, \xi / L) .
$$

Here $l$ is the linear extent of the avalanche (in the $d$ transverse directions; in the direction the interface moves, the extent is $\left.\sim l^{\zeta}\right), L$ is the linear size of the system, $\xi$ is the correlation length, and $z$ is the non-trivial dynamical exponent from the RG. For a fixed $l$ and $\xi$, as $L \rightarrow \infty$, the avalanche duration should be independent of $L$ since the system is uncorrelated on length scales much bigger than $\xi$ 24. It should also be independent of $\xi$, since requiring a total growth rate of $v L^{d}$ for the system does not affect any individual avalanche from proceeding with 
its own intrinsic timescale. Thus $T(0,0)$ is some non-zero constant.

In the other regime of $\xi>>L$, i.e. if the $v \rightarrow 0$ limit is taken before $L \rightarrow \infty$, the avalanches are non-overlapping in time. In the constant velocity driving mode, where the velocity constraint is imposed, any single avalanche proceeds at a fixed rate. Therefore for the constant velocity driving mode, $t(l, L, \xi)$ must be inversely proportional to the rate at which the avalanche is allowed to proceed, which is $v L^{d}$. Requiring that $t$ should have such a dependence on $v$ and $L$, using the result [3] $v \sim \xi^{\zeta-z}$, it is straightforward to verify that

$$
\lim _{\xi / L \rightarrow \infty} t(l, L, \xi) \sim l^{\zeta+d} /\left(v L^{d}\right) .
$$

This yields an apparent dynamical exponent of $z_{v}=\zeta+d$, which is the $d+1$ dimensional generalization of Eq.(3). Note that there is no change in the apparent dynamical exponent in the $\xi>>L$ regime for constant force driving, where an avalanche is allowed to proceed at its intrinsic rate (through parallel updating of lattice sites). This is why the RG estimate for $z$ for two dimensional CDWs agrees with the exact result for Abelian sandpiles [11].

The analysis of the interface roughness for a system of linear size $\mathrm{L}, w(t, L)$, proceeds in a similar manner. At a velocity $v$, the interface roughness has the scaling form

$$
w(t, L, \xi)=L^{\zeta} W\left(t / L^{z} ; \xi / L\right)
$$

For fixed $t$ and $\xi$, the roughness must have a well defined $L \rightarrow \infty$ limit:

$$
\lim _{L \rightarrow \infty} w(t, L, \xi)=\xi^{\zeta} W_{1}\left(t / \xi^{z}\right)
$$

For large $t$, the roughness saturates to the steady state form $w \sim \xi^{\zeta}$. For $\xi \rightarrow \infty$ (the large $L$ limit has been taken first), or equivalently for small $t$, the roughness is $\xi$ independent, i.e. $w \sim t^{\zeta / z}$.

In the other regime of $\xi>>L$, the apparent exponents are once again different for constant velocity driving. In this case, the dynamics are controlled by the imposed velocity, i.e. the $v$ and $t$ dependence of Eq.(9) occurs only in the combination $\tau=v L^{d} t$. Using $v \sim \xi^{\zeta-z}$, this implies

$$
\lim _{\xi / L \rightarrow \infty} w(t, L, \xi)=L^{\zeta} W_{2}\left(\tau / L^{\zeta+d}\right) .
$$

(With extremal dynamics, where one site moves forward at every time step, $\tau=t$.) In the large time limit, the roughness approaches the steady state form $w \sim L^{\zeta}$, i.e. $W_{2}(\infty)$ is a constant. It is not obvious how to extract the behavior of the function $W_{2}$ when its argument is small, but physical arguments supported by numerical results 13,16 show that $w(\tau, L)$ must have a residual $L^{-d / 2}$ dependence. Therefore

$$
w \sim\left(v L^{d} t\right)^{\beta v} / L^{-d / 2}
$$

with

$$
\beta_{v}=\frac{\zeta+d / 2}{\zeta+d}
$$

which is the same as Eq.(10) of Ref. [16] (generalized to $d$ dimensions).

Eqs.(8) and (12) give the $v \rightarrow 0$ scaling behavior of avalanche durations and interface roughness respectively for constant velocity driving. We note once again that these are for $\xi>>$, i.e. $v \rightarrow 0$ before $L \rightarrow \infty$. As mentioned before, even in critical phenomena for equilibrium phase transitions, as one approaches the transition for a fixed system size, one sees a change in the scaling form of dynamical variables. However, it is generally possible to obtain the behavior in this regime by requiring that there should be no dependence on (say) the reduced temperature $t$ for the behavior of a finite size system. This requirement comes from the fact that there are no thermodynamic singularities for a finite sized system: it is possible to go smoothly from one side of the phase transition to another. No such requirement exists for dynamical phase transitions, and one must be careful about possible $v$-dependence even in the $v \rightarrow 0$ regime [2].

There are other mechanisms as well that can make the connection between RG exponents and apparent scaling difficult. For instance, for CDWs below the depinning threshold, the periodicity of the dynamical variable (the CDW phase) prevents it from advancing by more than $2 \pi$ anywhere in a single avalanche, but the same periodicity makes a region that has just avalanched susceptible to an imminent 'retriggering' of a fresh avalanche [27. The low frequency dynamics thus sees a non-trivial analog of the roughness exponent, and the distribution of avalanche sizes is singular as $v \rightarrow 0$. Another example is for interface roughness itself, where the steady state roughness over a subsystem of size $x$ in a system with size $L$ scales as $w^{2}(x ; L) \sim x^{2} L^{2 \zeta-2}$ when $\zeta>1$ [28]. This is because the Fourier transform of the steady state roughness must be well behaved as $L \rightarrow \infty$, and for $\zeta>1 w^{2}(x ; L)$ is dominated by long wavelength modes with $q \sim 1 / L$.

In this paper we have shown that the renormalization group flows for depinning transitions are the same whether the system is driven with a constant force or a constant velocity. However, in the critical regime, the apparent scaling behavior of physical quantities can be different for the two driving modes. This is because scaling functions can depend on the system velocity in a singular manner as the transition is approached. This is unlike what is seen in equilibrium critical phenomena, and seems to be more common in the constant velocity driving mode. Despite this caveat, it is possible to obtain results for one driving mode from the other.

I thank Anne Tanguy and Maya Paczuski for useful discussions. 
[1] D.S. Fisher, Phys. Rev. Lett. 50, 1486 (1983); D.S. Fisher, Phys. Rev. B 31, 1396 (1985).

[2] O. Narayan and D.S. Fisher, Phys. Rev. Lett. 68, 3615 (1992); Phys. Rev. B 46, 11520 (1992).

[3] T. Nattermann, S. Stepanow, L-H. Tang and H. Leschhorn, J. Phys. (France) II 2, 1483 (1992); O. Narayan and D.S. Fisher, Phys. Rev. B 48, 7030 (1993).

[4] D. Ertas and M. Kardar, Phys. Rev. E 49, 2532 (1994); J.P. Sethna and K. Dahmen, Phys. Rev. Lett. 71, 3222 (1993) and Phys. Rev. B 53, 14872 (1996); L. Balents, M.C. Marchetti and L. Radzihovsky, Phys. Rev. B 57, 7705 (1998); P. Chauve, T. Giamarchi and P. le Doussal, Europhys. Lett. 44, 110 (1998) and cond-mat/0002299.

[5] P. Bak, C. Tang and K. Wiesenfeld, Phys. Rev. Lett. 59, 381 (1987).

[6] M. Paczuski, S. Maslov and P. Bak, Phys. Rev. E 53, 414 (1996) and references therein.

[7] C. Tang and P. Bak, Phys. Rev. Lett. 60, 2347 (1988); C. Tang, K. Wiesenfeld, P. Bak, S.N. Coppersmith and P.B. Littlewood, Phys. Rev. Lett. 58, 1161 (1987); S.N. Coppersmith and P.B. Littlewood, Phys. Rev. B 36, 311 (1987).

[8] O. Narayan and A.A. Middleton, Phys. Rev. B 49, 244 (1994).

[9] The mapping [8] is from a CDW with periodic boundary conditions to a sandpile [5] with periodic boundary conditions, instead of the standard open boundary conditions. This creates an 'above threshold' phase for the sandpile, where grains tumble around for ever without additional injection. However, avalanches below threshold are unaffected for a sufficiently large system. Another difference is that ramping up the force below threshold for a CDW corresponds to dropping sand grains on all the sites one by one in a random order, and cycling through this sequence repeatedly, instead of the standard spatially and temporally random dropping of sand. Once again, this does not matter in the large system limit.

[10] M. Paczuski and S. Boettner, Phys. Rev. Lett. 77, 111 (1996). The mapping is from a SOC model to a $1+1$ dimensional interface driven by pulling it at one end at a constant speed. This is equivalent (after averaging over randomness) to driving the interface with a uniform applied force, with one end constrained to move at the (spatial and temporal) average velocity of the entire interface. The constraint is inconsequential for large systems.

[11] S. Majumdar and D. Dhar, Physica A 185, 129 (1992).

[12] K. Sneppen, Phys. Rev. Lett. 69, 3539 (1992). Only model $\mathrm{B}$ of this paper will be considered.

[13] H. Leschhorn and L-H. Tang, Phys. Rev. E 49, 1238 (1994).

[14] A. Tanguy, M. Gounelle and S. Roux, Phys. Rev. E 58, 1577 (1998). This paper considers long ranged as well as (effectively) short ranged elastic interactions.

[15] K. Sneppen and M.H. Jensen, Phys. Rev. Lett. 71, 101 (1993).

[16] S. Krishnamurthy, A. Tanguy and S. Roux, Eur. Phys. J. B 15, 149 (2000).

[17] In Ref. [16], using the model of Ref. 14], the interface is in fact started with a flat configuration, but the same results are obtained starting from steady state (A. Tanguy, private communication). For the model of Ref. [12], these results are only obtained starting from steady state [15]. This is probably because the model of Ref. 12 is a 'hybrid' model, where only a single site moves at a time step, but can then trigger a brief avalanche (at the same time step). This is neither constant velocity nor constant force driving. In steady state, the avalanches have a characteristic size, and the model effectively has constant velocity driving. However, when one starts from a flat interface, the mean avalanche size rises from unity to its saturated value; in fact, all scaling in the short time regime for this case is quite imperfect. (The connection between the changing mean avalanche size and the peculiar short time behavior of the roughness is seen better in the original version of the model [12], where the initial pinning strengths are Gaussian distributed, but the new pinning strengths when sites move are uniform over [0,1).) Also, concepts of correlation lengths and compact avalanches are more tenuous 13 in this model.

[18] The scaling form looks slightly different than that in Ref. [16], because the time $t$ is used and not the scaled time $\theta$. The dynamic exponent $z_{v}$ is the $z_{2}$ of Ref. 16.

[19] Numerical estimates $z_{v}$ and $\zeta$ for interfaces with short range elasticity (see Eq.(1) ) in 1+1 dimension violate this equation, but this is probably because of pathologies for $\zeta>1$. Numerical estimates for long range elastic models in $1+1$ dimension satisfy this equation 14.

[20] R. Bruinsma and G. Aeppli, Phys. Rev. Lett. 52, 1547 (1984); J. Koplik and H. Levine, Phys. Rev. B 32, 280 (1985); D.A. Kessler, H. Levine and Y. Tu, Phys. Rev. A 43, 4551 (1991).

[21] M. Kardar, G. Parisi and Y-C. Zhang, Phys. Rev. Lett. 56, 889 (1986). Such terms exist and are relevant for an interface moving in an anisotropic medium, where the pinning has different strength in different directions [22].

[22] L.A.N. Amaral, A.-L. Barabasi and H.E. Stanley, Phys. Rev. Lett. 73, 62 (1994); L-H. Tang, M. Kardar and D. Dhar, Phys. Rev. Lett. bf 74, 920 (1995).

[23] P.C. Martin, E. Siggia and H. Rose, Phys. Rev. A 8, 423 (1973); H.K. Janssen, Z. Phys. B 23, 377 (1976).

[24] Avalanches can actually not be clearly separated in this regime, but it may be possible for continuum models to do this approximately by separating out fast and slow motion. In automaton models, this is much more problematic 25], but in any case, the discussion here should be valid for time scales corresponding to length scales for any measure of activity.

[25] A. Corral and M. Paczuski, Phys. Rev. Lett. 83, 572 (1999). For the model of Ref. [10], the avalanches are found to merge into each other for strong driving. The scale found for the crossover from separate to merged avalanches is in fact just what one would expect by requiring $\xi \sim L$ and using numerically measured exponents for $1+1$ dimensional depinning: $x=1 /(z-\zeta)=$ $0.205 \pm 0.015$, compared to 26] $\zeta=1.25 \pm 0.01$ and $\zeta / z=0.88 \pm 0.02$. Other exponents in this paper also agree with results for $1+1$ dimensional depinning [26].

[26] H. Leschhorn, Physica A 195, 324 (1993).

[27] A.A. Middleton, Ph.D. thesis, Princeton University, 1990; see also Ref. [8].

[28] H. Leschhorn and L-H. Tang, Phys. Rev. Lett. 70, 2973 (1993). 